\title{
CORRELATION BETWEEN PHENOTYPIC AND GENOTYPIC IDENTIFICATION OF
} TRICHOPHYTON VIOLACEUM

\author{
Ali Soliman, Samia Ali Ibrahim, Mohamed Taha* and Ayman Farouk \\ Departments of Dermatology and Microbiology* \\ Faculty of Medicine and Veterinary Medicine, Zagazig University
}

\begin{abstract}
Background: Trichophyton violaceum is an anthropophilic dermatophyte. It is the most common etiologic agent causing dermatophytosis especially tinea capitis in Egypt. As routine phenotypic identification of dermatophytes is either slow or lack specificity, improved identification methods are required. The application of PCR has made rapid and precise identification of $T$. violaceum possible.

Aim: This study was designed to correlate between phenotypic and genotypic identification of $T$. violaceum. Subjects: Two hundred patients with tinea capitis (122 males and 78 females) with a mean age of $8.5 \pm 3.1$ were the subjects of this study.

Methods: Phenotypic identification of $T$. violaceum determined by observation of its macroscopic and microscopic characteristics and culture on differential media was correlated with ITS-based PCR.

Results: Genotypic identification results of $T$. violaceum in comparison with phenotypic identification results were identical.

Conclusion: Although there is no difference between the results of phenotypic and genotypic identification methods of $T$. violaceum isolates, genotypic method is recommended as it is rapid and accurate.

Keywords: Trichophyton violaceum, identification, phenotypic, dermatophytes, PCR and genotypic.
\end{abstract}

\section{INTRODUCTION}

D ermatophytosis, commonly referred to as ringworm, is a superficial fungal infection caused by filamentous fungal species (dermatophytes) belonging to three anamorphic genera, Trichophyton, Microsporum, and Epidermophyton. It is the most common superficial fungal infection in the world. Dermatophytes are a group of closely related keratinophilic fungi that have the ability to infect the keratinized tissues (skin, hair, and nails) of humans and animals ${ }^{1}$. Members of the genus Trichophyton are the commonest agents of dermatophytoses ${ }^{2}$.

Classical diagnosis of dermatophytosis consists of direct microscopy and culture with subsequent species identification mainly based on macroscopic and microscopic features of the culture. Molecular techniques are increasingly being employed in the clinical microbiological laboratory for identification because of the high sensitivity, specificity and speed ${ }^{3}$.

Trichophyton violaceum is an anthropophilic dermatophyte. It mainly causes tinea capitis and to a lesser extent tinea corporis, tinea pedis, tinea facei and tinea unguium. It is common in Middle East and North Africa. In Egypt it is the most common etiologic agent causing dermatophytosis especially tinea capitis ${ }^{4-}$ 5 .

Gräser et al in $2008^{6}$ described three prominent phenotypes of $T$. violaceum : (1) classic pan-African and west/central Asian T. violaceum, consisting of dense, slow-growing and glabrous (this combination of characters is called "faviform"), predominantly blood-red colonies, sometimes with whitish sectors, and with a uniform stimulation response to thiamine; (2) isolates corresponding to the $T$. violaceum synonym " $T$. glabrum," similar to typical $T$. violaceum except whitish in color, mostly coming from the Horn of Africa region (Eritrea and Somalia); and (3) central African isolates corresponding to the synonym " $T$. yaoundei" which are faviform and whitish but often secrete a brown pigment into surrounding Sabouraud glucose agar.

Trichophyton violaceum microscopically usually lack sporulation although variants with macroconidida and microconidia have been described ${ }^{7}$.

Trichophyton glabrum, T. endicum, T. yaoundei, T. gourvilii, T. Soudanense are synonyms for $T$. violaceum. All $T$. violaceum lineages show an identical effect on Bromocresol purple (BCP)-milk solids- glucose agar that appears as a wide zone of clearing around the colonies, with some alkalinization of the medium ${ }^{6}$.

\section{AIM OF THE WORK}

To correlate between phenotypic and genotypic identification of $T$. violaceum.

\section{SUBJECTS}

Two hundred patients with tinea capitis presented to the Dermatology Department of Zagazig University Hospitals during the period from December 2011 to April 2013 were the subjects of this study. The diagnosis of tinea 
capitis was established clinically.

\section{METHODS}

Skin scrapings and hair fragments were collected from all cases and subjected to direct microscopy by $\mathrm{KOH}(20 \%)$ and culture on Sabouraud's dextrose agar (SDA) with chloramphenicol and cycloheximide. After incubation at $25^{\circ} \mathrm{C}$ for two weeks the isolates of $T$. violaceum were subjected to identification by the following methods:

A. Phenotypic method. Phenotypic identification of the isolates was performed through macromorplological, micromorphological examination of colonies and culture on Dermatophyte Test Media (DTM) ${ }^{\mathbf{8}}$, Rice Lactritmal Agar (RLA) 9 , BCP-milk solidsdextrose agar ${ }^{\mathbf{1 0}}$ and Milk honey bromothymol blue (MHB) ${ }^{11}$.

B. Genotypic method. Twenty eight isolates of $T$. violaceum were subjected to genotypic identification through the following:

1. DNA extraction. A small part each culture growth was taken and transferred to sterile prechilled mortars and ground finely with a pestle after addition of liquid nitrogen. The resulting powder was aliquoted into sterile, $1.5-\mathrm{ml}$ microcentrifuge tubes. The powder specimens that could not be processed immediately were frozen at $-20^{\circ} \mathrm{C}$. Trichophyton violaceum DNA was extracted using the organic phenol/ chloroform method according to the protocol of Sambrook and Russel 12 and the spin column method using Genomic DNA mini extraction kit (Applied biotechnology co. Egypt) according to Ahmed ${ }^{\mathbf{1 3}}$ for comparison between both methods on 7 isolates.

2. Amplification. Amplification reactions were carried out with $1 \mathrm{X}$ of Master mix (BioTeke Corporation, China), $25 \mathrm{pmol}$ each of primers ITS1 [5'-TCCGTAGGTGAACCTGCGG-3'] and ITS4 [5'-TCCTC CGCTTATTGATATGC-3'] (Alpha DNA, Canada after White et al. ${ }^{\mathbf{1 4}}$ ), $100 \mathrm{ng}$ of DNA extract and nuclease free water up to a total volume of $50 \mu \mathrm{L})$. Amplification was performed with a PCR thermal cycler (Master cycler Pro S, Epedndorf, Germany) using initial denaturation at $95^{\circ} \mathrm{C}$ for $5 \mathrm{~min}$, followed by 30 cycles at $95^{\circ} \mathrm{C}$ for $30 \mathrm{sec}, 56^{\circ} \mathrm{C}$ for $30 \mathrm{sec}$, and $72^{\circ} \mathrm{C}$ for $30 \mathrm{sec}$ with final extension at $72^{\circ} \mathrm{C}$ for 7 $\min$.

3. Visualization of PCR products by agarose gel electrophoresis. The resulting products were separated in $2 \%$ agarose gels and $1 \mathrm{X}$ Tris-acetateEDTA buffer and stained with ethidium bromide. Using UV Transilluminator the amplified product was photographed and image analysis was done by Gel documentation system.

\section{Statistical analysis:}

Data were checked, entered and analyzed using (SPSS version 20). Data were expressed as mean \pm SD for quantitative variables, number and percentage for categorical variables. Chi-Square test $\left(\mathrm{X}^{2}\right)$ or Fisher exact results were used when appropriate. $\mathrm{P}<0.05$ was considered statistically significant.

\section{RESULTS}

Among the 200 clinically diagnosed cases as tinea capitis [aged 4-12 years with a mean of 8 .

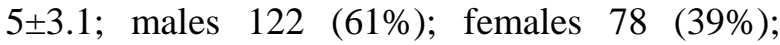
duration of symptoms ranged from 3 days to 5 months; positive family history in $67 \%$ and positive animal contact history in $79 \%$ ] the most common clinical type was the scaly $(60 \%)$, the next common type was the black dot $(39 \%)$, while the third type was the kerion $(1 \%)$.

Direct microscopy revealed fungal elements in hairs and/or scales in all 200 cases. Out of the $200 \mathrm{KOH}$ positive samples cultured on SDA, 164 cases $(82 \%)$ yielded positive dermatophyte culture, while 36 cases (18\%) were negative.

Trichophyton violaceum was the commonest species responsible for tinea capitis as it was isolated from $69 \%$ of cases. While T.violaceum was isolated from $56.6 \%$ of cases of scaly type of tinea capitis, it was isolated from $89.7 \%$ cases of black dot type of tinea capitis.

The macromorphology of isolated T.violaceum revealed glabrous and waxy colonies that showed variation in the pattern of growth (limited with small colonies and diffuse with large colonies) and the color (red to dark red and violet).

The micromorphology of isolated T.violaceum revealed variation as follows: septated branched hyphae, septated branched hyphea with few microconidia and chlamydospores, bizare hyphea with chamydospores and chains of chamydospores.

While T.violaceum grew well on DTM and turned it red (Fig.1), its pigment was stimulated and appeared more reddish with slow penetration on RLA (Fig.2).

On (BCP)-milk solids-dextrose agar medium a hallow clear zone around $T$. violaceum colonies was detected after 10 days (Fig.3) and color of the medium turned to faint purple after 14 days (Fig.4), but after more than 14 days $T$. violaceum showed profuse growth with change of the color of the medium (Fig.5).

Milk honey bromothyoml blue medium after 4 days showed zone of casein hydrolysis before growth detection (Fig.6), but after 10 days 
showed profuse growth and green color around $T$. violaceum colonies (Fig.7).

In this study molecular examination was done for 28 isolates of $T$. violaceum by ITS-based PCR to confirm their identification by the phenotypic method.

DNA extraction using the classic method phenol/chloroform method was successful in contrast to the spin column method during the comparison between both methods on 7 isolates (Fig.8, $9 \& 10$ ).

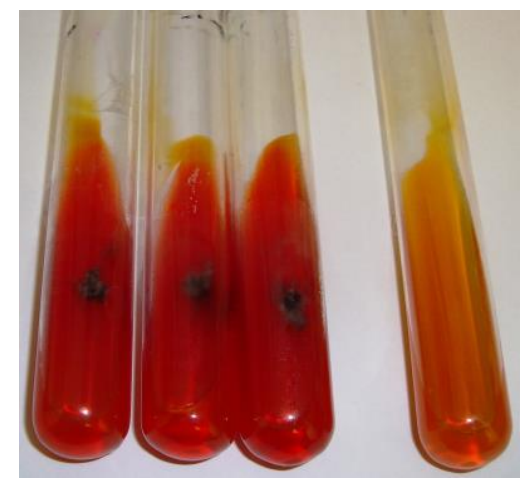

Fig. (1): T. violaceum on DTM: colonies growing well and turning the color of medium to red (10 days).
In the present study PCR-based identification using PCR with the universal ITS1/ITS4 primer set was performed. This primer set amplified the ITS I, 5.8S, and ITS II regions of the ribosomal DNA in all 28 tested isolates (7 isolates during the comparison between both methods of DNA extraction and other 21 isolates), resulting in amplified products (expected bands) of approximately $690 \mathrm{bp}$ of the DNA ladder (Fig. $8,9,10,11,12)$.

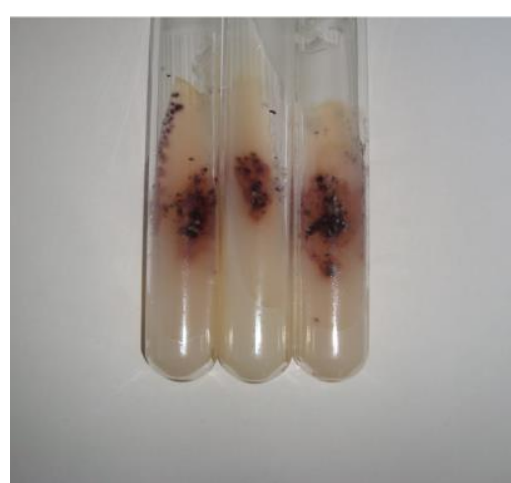

Fig. (2): T.violaceum on RLA: profuse growth and diffuse red pigmentation in the medium.

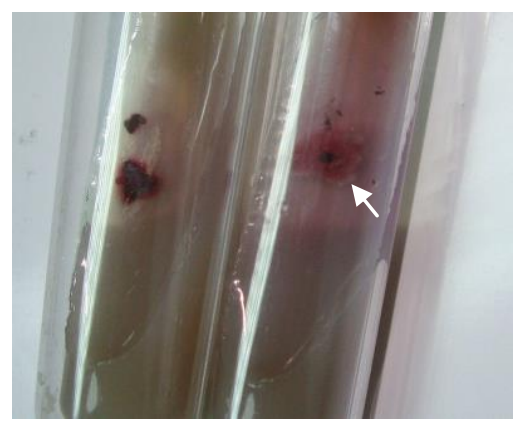

Fig. (3): T. violaceum on (BCP)-milk solids-dextrose agar after 10 days: clear zone around the colonies.

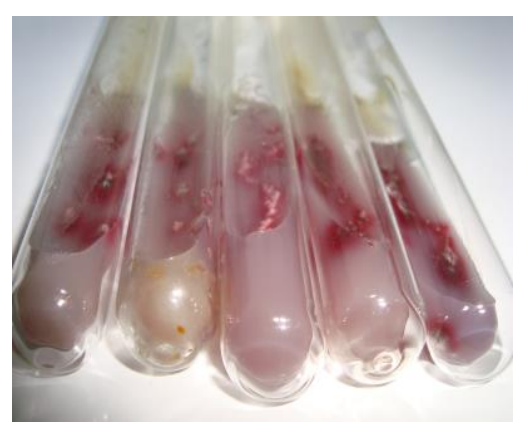

Fig. (4): T. violaceum on (BCP)-milk solids-dextrose agar after 14 days: faint purple color.

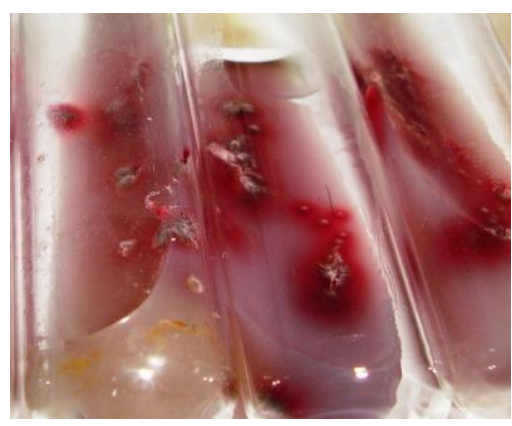

Fig. (5): $T$. violaceum on (BCP)milk solids-dextrose agar after more than 14 days: profuse growth with diffuse red pigment and slight change in the medium color. 


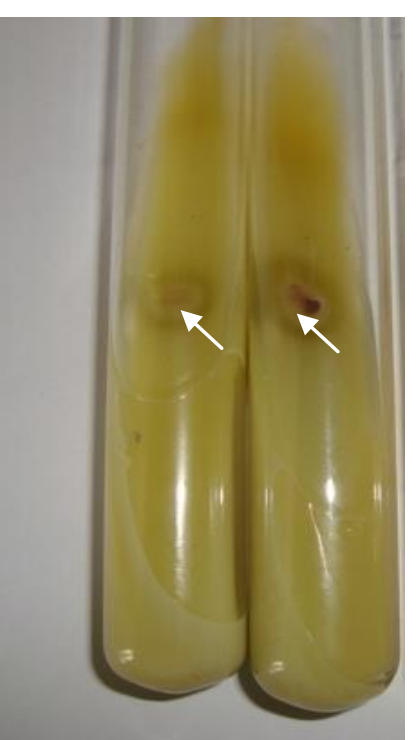

Fig. (6): T. violaceum on MHB: zone of casein hydrolysis and before appearance of growth (4days).

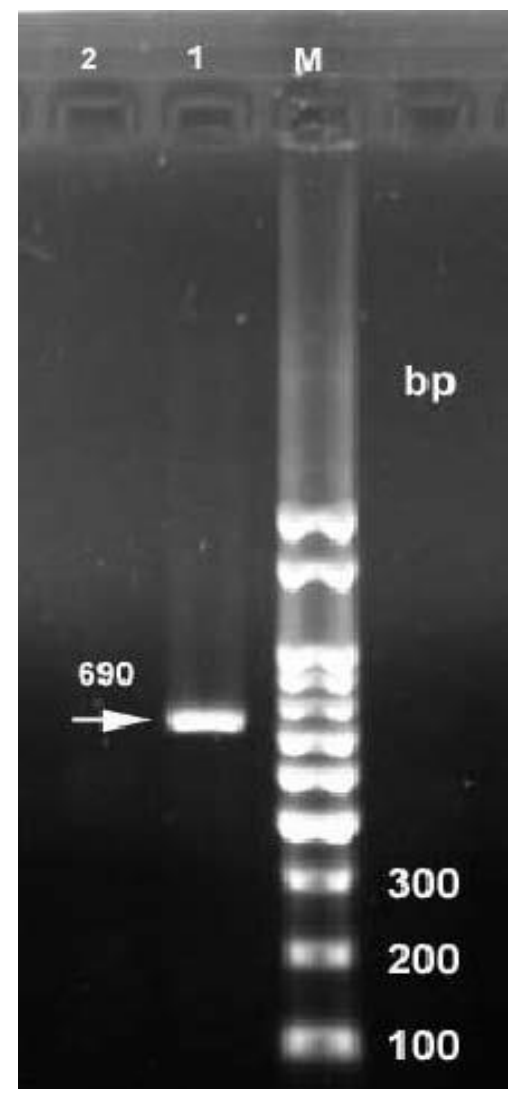

Fig. (8): Agarose gel electrophoresis: PCR products of the ITS I, 5.8S, and ITS II regions of one phenotypically identified sample.

M: 100 bp DNA ladder (Molecular Marker).

Lane 1: Using phenol chloroform method the sample showed positive result (690 bp).

Lane 2: Using spin column method the same
Fig. (7): T. violaceum on MHB: profuse growth after 10 days with zone of casein hydrolysis and green color around the colonies.

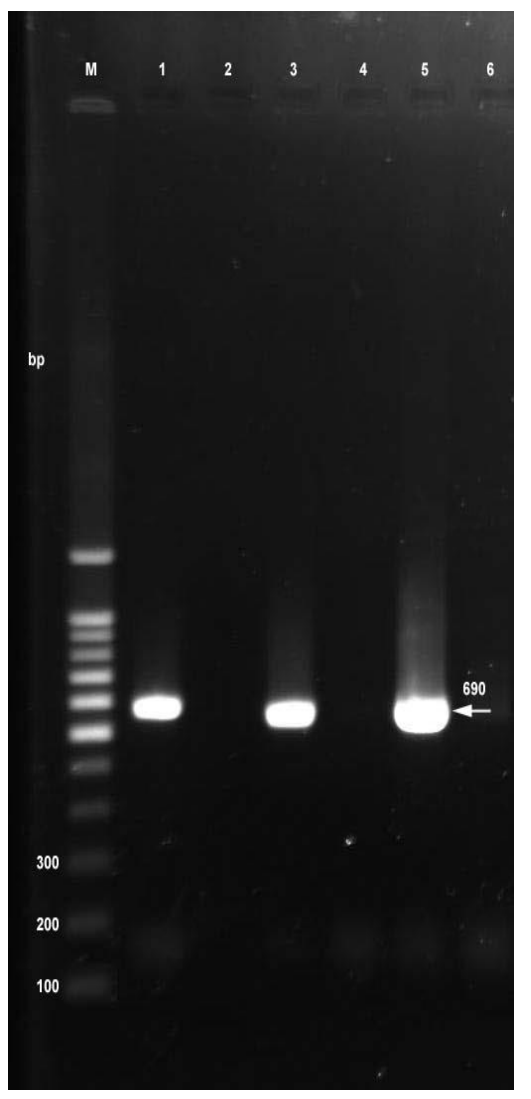

Fig. (9): Agarose gel electrophoresis: PCR products of the ITS I, 5.8S, and ITS II regions of 3 phenotypically identified species.

M: 100 bp DNA ladder (Molecular Marker).

Lane 1, 3 and 5: Using phenol chloroform method 3 samples showed positive result (690 bp).

Lane 2, 4 and 6: Using spin column method the same 
sample showed negative result. 3 samples showed negative result.

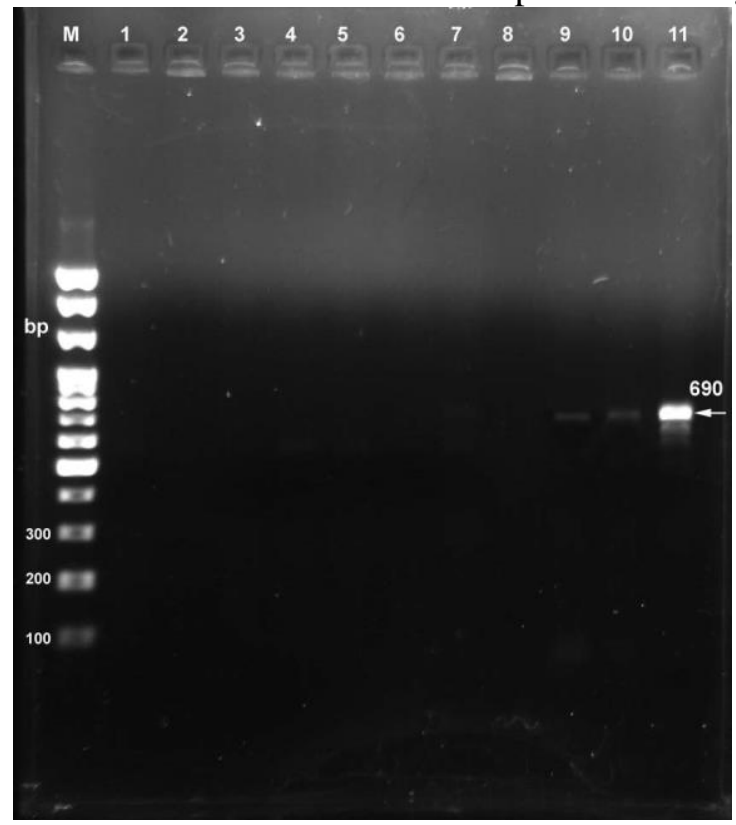

Fig. (10): Agarose gel electrophoresis: PCR products of the ITS I, 5.8S, and ITS II regions of 3 phenotypically identified species.

M: 100 bp DNA ladder (Molecular Marker).

Lane: 9, 10 and11: Using phenol chloroform method 3 samples showed positive result (690 bp).

Lane: 6, 7 and 8: Using spin column method the same 3 samples showed negative result.

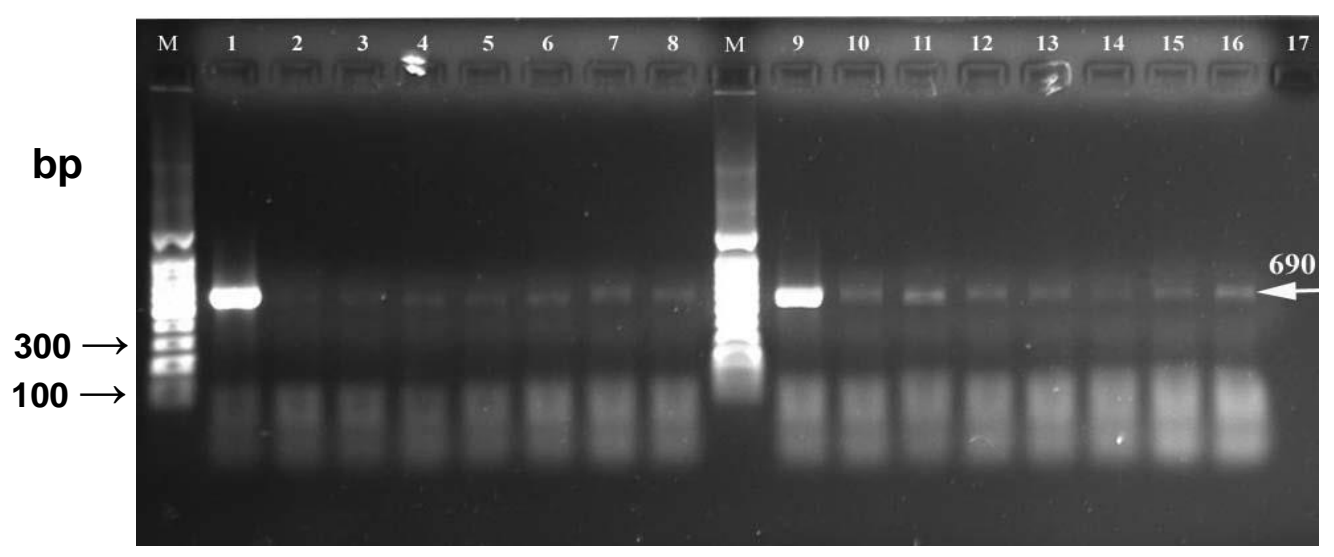

Fig. (11): Agarose gel electrophoresis: PCR products of the ITS I, 5.8S, and ITS II regions of 15 phenotypically identified species. M: 100 bp DNA ladder (Molecular Marker).

Lane 1: Positive control (690 bp).

Lane 17: Negative control.

Lane 2, 3, 4, 5, 6, 7, 8, 9, 10, 11, 12, 13, 14, 15 and 16: 15 positive cases (690 bp). 


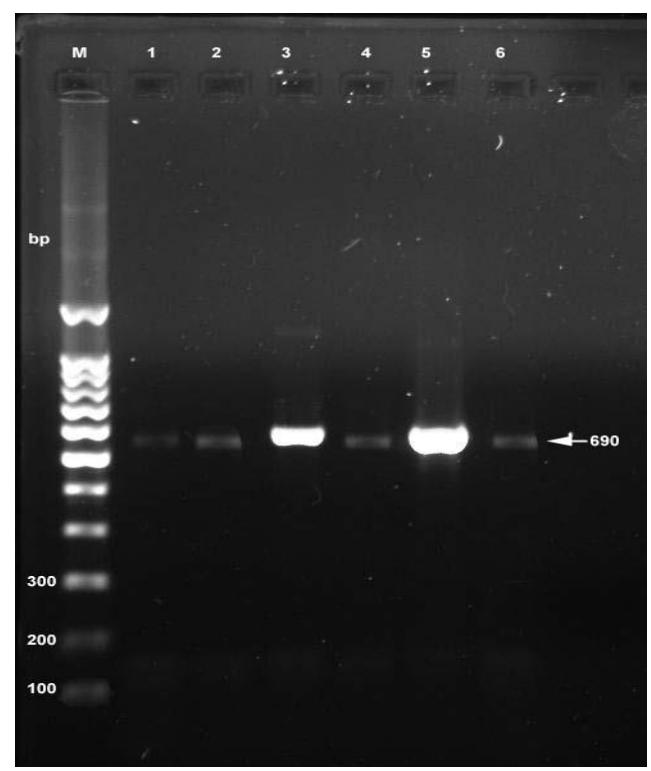

Fig. (12): Agarose gel electrophoresis: PCR products of the ITS I, 5.8S, and ITS II regions of 6 phenotypically identified species.

M: $100 \mathrm{bp}$ DNA ladder (Molecular Marker).

Lane 1, 2, 3, 4, 5 and 6: 6 positive cases (690 bp).

\section{DISCUSSION}

Dermatophytes are a group of closely related keratinophilic fungi that can invade keratinised humans and animal's tissues such as skin, hair and nails causing dermatophytosis ${ }^{15}$. The etiologic agents of dermatophytosis are classified into three genera based primarily on differences in microscopic morphology and modes of sporulation as Epidermophyton, Mirosporum and Trichophyton ${ }^{16}$.

Tinea capitis is a highly contagious common dematophytic infection seen predominantly in children caused predominantly by Trichophyton or Microsporum species ${ }^{17}$.

Clinical presentation of disease revealed that scaly type to be the commonest (60\%) followed by black dot type (39\%) and kerion type was the least (1\%).These findings in this study were similar to the study of Gargoom et al. ${ }^{18}$ and Jha et al. ${ }^{19}$.

Although all of our cases were $\mathrm{KOH}$ Positive $(100 \%)$ but only (82\%) of them were culture positive and the remaining (18\%) showed no growth as reported by Zaraa et al. ${ }^{20}$.

In the present study the causative agents of tinea capitis were dominated by two species $T$. violaceum that was recovered from 138 cases (69\%) and M. canis from 26 cases (13\%). These findings similar to other studies done by Gargoom et al. ${ }^{\mathbf{1 8}}$ and Zaki et al. ${ }^{\mathbf{2 1}}$.

In our study $T$. violaceum isolated from $(56.6 \%)$ of cases of scaly type of tinea capitis, while $(89.7 \%)$ of cases of black dot type gave
T.violaceum on culture. This result coincides with the study done by Amer et al. ${ }^{4}$.

Phenotypic identification of T.violaceum in this work for 200 isolates obtained from cases of tinea capitis relied on macromorphology, micromorphology and culture on four differential media namely DTM, RLA, BCP-milk solidsdextrose agar and MHB.

Concerning the macromorphology and micromorpholgy of T.violaceum in this study it was found that all isolates showed typical characteristics as reported by Larone ${ }^{22}$ and Padhye \& Summerbell ${ }^{23}$.

While all isolates of T.violaceum in the present work grow well on DTM and turned it red in agreement with Taplin et al. ${ }^{8}$, Lactritmel agar enhanced the sporulation and increased the production of pigment in agreement with Aboul Magd and Taha ${ }^{9}$. They added that this media is one of the most widely used differential media.

The use of BCP-milk solids-dextrose agar facilitated the confirmatory identification of T.violaceum that produced a clear zone around its colonies and changed the color of the medium to purple slowly. These results of the current study were found to be the same as reported by Khafagy $\&$ Taha ${ }^{10}$.

In our work identification of T.violaceum by MHB showed profuse growth with zone of casin hydrolysis and green color around the colonies as findings mentioned by Taha et al. ${ }^{\mathbf{1 1}}$.

The conventional methods to identify dermatophytes rely on the expression of characteristic phenotypic characters. Identification 
is often delayed or problematic because isolates may be slow to form conidia or produce atypical microscopic structures or colony appearances that require the assistance of an experienced mycologist who specializes in dermatophytes ${ }^{24}$.

Molecular techniques are more beneficial for dermatophyte identification as they are objective, unequivocal, rapid and more sensitive. Moreover, these methods rely on genetic makeup, which is more constant than phenotypic characterization, and they can identify atypical dermatophytes that could not be identified by culture-based techniques ${ }^{\mathbf{2 4}}$.

Analysis of the ITS regions of ribosomal DNA is a simple and reproducible molecular tool for identification of $T$. violaceum. To determine the correlation between PCR-based methods with phenotypic techniques, we performed PCR-based identification using PCR with the universal ITS1/ITS4 primer set. This primer set amplified the ITS I, 5.8S, and ITS II regions of the ribosomal DNA in all 28 phenotypically identified $T$. violaceum tested isolates, resulting in amplified products of approximately $690 \mathrm{bp}$ which means successful amplification of the DNA. Similar results obtained by Shehata et al. ${ }^{25}$.

Concerning with DNA extraction for isolates of $T$. violaceum two methods are compared in this work: the classic method with phenol/chloroform and that with spin column. Extraction products underwent ITS-based PCR to find the best method of DNA extraction from $T$. violaceum. After determination of the genomic profiles obtained from the DNA extracted from the same isolates with Phenol/Chloroform method and not with spin column method, we concluded that the extraction method with Phenol/Chloroform from $T$. violaceum cells is better than with spin column and greatly facilitating the obtainment of $T$. violaceum DNA. The failure of spin column method in the current study was in contrast to results of study of Ahmed. ${ }^{\mathbf{1 3}}$ who had succeeded in extracting DNA from Microsporum canis by this method and this may be attributed to increased pigmentation in case of $T$. violaceum or difference in the ultrastructure of cell wall between $T$. violaceum and Microsporum canis.

In this work, we successfully identified the tested isolates to the species level in full agreement with both the phenotypic and the genotypic (ITS-based PCR) methods. Similar findings reported by Shehata et al. ${ }^{25}$.

In conclusion, although genotypic identification results of $T$. violaceum in comparison to phenotypic identification results were identical, we recommended the use of genotypic method for rapid and accurate result especially in isolates showing atypical morphological characters.

\section{REFERENCES}

1. Kanbe T (2008): Molecular approaches in the diagnosis of dermatophytosis. Mycopathologia; 166(5-6):307-17.

2. Kong F, Tong Z, Chen X et al (2008): Rapid identification and differentiation of Trichophyton species, based on sequence polymorphisms of the ribosomal internal transcribed spacer regions, by rolling-circle amplification. J Clin Microbiol; 46(4):11929.

3. Wisselink GJ, van Zanten E and KooistraSmid AM (2011): Trapped in keratin; a comparison of dermatophyte detection in nail, skin and hair samples directly from clinical samples using culture and real-time PCR. J Microbiol Methods; 85(1):62-6.

4. Amer M, Taha M, Tosson Z et al (1981): The frequency of causative dermatophytes in Egypt. Int J Dermatol; 20(6): 431-4.

5. Omar AA (2000): Ringworm of the scalp in primary-school children in Alexandria: infection and carriage. East Mediterr Health J; 6(5-6):961-7.

6. Gräser Y, Scott J and Summerbell R (2008): The new species concept in dermatophytes-a polyphasic approach. Mycopathologia; 166(5-6): 239-56.

7. Magill SS, Manfredi L, Swiderski A et al (2007): Isolation of Trichophyton violaceum and Trichophyton soudanense in Baltimore, Maryland. J Clin Microbiol; 45(2):461-5.

8. Taplin D, Zaias N, Rebell G et al (1969): Isolation and recognition of dermatophytes on a new medium (DTM). Arch Dermatol; 99:203-9.

9. Aboul magd $\mathrm{M}$ and Taha M (2001): Rice Lactritmel Agar: A new modification of Borelli's medium for identification of dermatophytes. Egypt J Med Microbiol; 10:595-605.

10. Khafagy $\mathrm{N}$ and Taha M (2003): Evaluation of Bromocresol purple media for identification of fungi isolated from the skin and nail infections. J Med Microbiol; 12:359-368.

11. Taha M, Elfangary M and Saudy W (2013): MHB: a new medium for isolation and identification of dermatophytes. J E W D S; 10 (3):172-176.

12. Sambrook J and Russell D W (2001): Molecular Cloning: a Laboratory Manual. 
3rd ed. New York: Cold Spring Harbor Laboratory Press.

13. Ahmed A (2013): Studies on Microsprum canis isolated from humans and animals. B.V.Sc., thesis, Fac. Vet. Med., Zagazig Univ.

14. White $T$, Bruns $T$, Lee $S$ et al (1990): Amplification and direct sequencing of fungal ribososmal RNA genes for phylogenetics. In: Innis M, Gelfand D, Sninsky J, White T, editors. PCR protocols. New York, NY: Academic Press, Inc; p. $315-22$.

15. Garg J, Tilak R, Garg A et al (2009): Rapid detection of dermatophytes from skin and hair. BMC Res Notes; 18; 2: 60.

16. Madhavi S, Rama Rao MV and Jyothsna K (2011):Mycological study of Dermatophytosis in rural population. Annals of Biological Research; 2(3):88-93.

17. Grover C, Arora P and Manchanda V. Tinea capitis in the pediatric population (2010): a study from North India. Indian J Dermatol Venereol Leprol; 76(5):527-32.

18. Gargoom AM, Elyazachi MB, Al-Ani SM et al (2000): Tinea capitis in Benghazi, Libya. Int J Dermatol; 39(4):263-5.
19. Jha BN, Garg VK, Agrawal S et al (2006): Tinea capitis in eastern Nepal. Int J Dermatol; 45(2):100-2.

20. Zaraa I, Hawilo A, Trojjet S et al (2012): Letter: Tinea capitis in infants in their first 2 years of life: A 12-year study and a review of the literature. Dermatol Online J; 18(7):16.

21. Zaki SM, Ibrahim N, Aoyama K et al (2009): Dermatophyte infections in Cairo, Egypt. Mycopathologia; 167(3):133-7.

22. Larone DH (2002): Medically important fungi: a guide to identification, 4th ed. ASM Press, Washington, DC.

23. Padhye A A and Summerbell R C (2005): The dermatophytes. In W. G. Merz and R. J. Hay (eds): Topley and Wilson's microbiology and microbial infections: medical mycology, 10th ed. ASM Press, Washington, DC, P. 220-243.

24. Gräser Y, el Fari M, Presber W et al (1998): Identification of common dermatophytes (Trichophyton, Microsporum, Epidermophyton) using polymerase chain reactions. $\mathrm{Br} \mathrm{J}$ Dermatol; 138(4):576-82.

25. Shehata AS, Mukherjee PK, Aboulatta $\mathrm{HN}$ et al (2008): Single-step PCR using (GACA) 4 primer: utility for rapid identification of dermatophyte species and strains. J Clin Microbiol; 46(8):2641-5. 


\section{الارتباط بين التصنيف المظهري والجيني}

للتريكوفيتون فيوليشيم النظري واليفر

إن التريكو فيتون فيوليشيوم هي أكثر أنواع الفطريات المسبية لسعفة الرأس، و قد كانت تصنف بالطرق المورفولوجية التقليديـة ولكن

مقدمة:

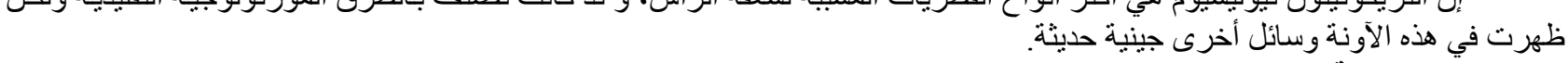

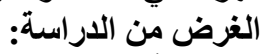

أجريت هذه الدر اسة للربط بيين الخو اص المورفولوجية و الجينية للتريكو فيتون فيوليشيوم.

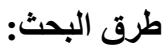

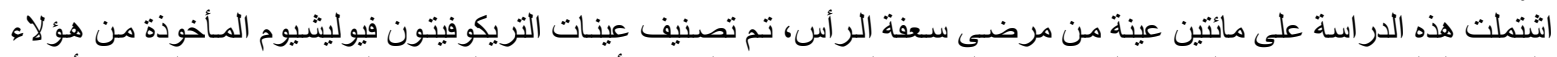

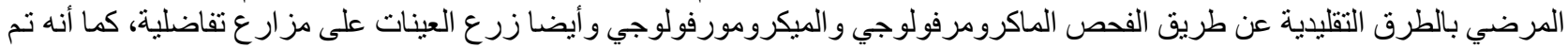
تصنيف مجمو عة من هذه العينات باستخدام تفاعل البلمرة المتسلسل.

$$
\begin{aligned}
& \text { وجد أن نتائج التصنيف الجيني مطابقة لنتائج التصنيف المظهري. } \\
& \text { النتائج: } \\
& \text { التصنيف الجيني للتريكو فيتون فيوليشيوم أدق و أسر ع من التصنيف المظهري. }
\end{aligned}
$$

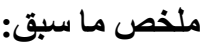

ننصح باستخدام التصنيف الجيني للتريكو فيتون فيوليشيوم كبديل للتصنيف المظهري حيث أنه أدق و أسرع. 